\title{
Function-based hybrid synchronization types and their coexistence in non-identical fractional-order chaotic systems
}

\author{
Adel Ouannas ${ }^{1}$, Giuseppe Grassi ${ }^{2}$, Xiong Wang ${ }^{3}$, Toufik Ziar ${ }^{4}$ and Viet-Thanh Pham ${ }^{5 *}$
}

"Correspondence:

phamvietthanh@tdt.edu.vn

${ }^{5}$ Modeling Evolutionary Algorithms

Simulation and Artificial

Intelligence, Faculty of Electrical \&

Electronics Engineering, Ton Duc

Thang University, Ho Chi Minh City,

Vietnam

Full list of author information is

available at the end of the article

\begin{abstract}
This paper presents new results related to the coexistence of function-based hybrid synchronization types between non-identical incommensurate fractional-order systems characterized by different dimensions and orders. Specifically, a new theorem is illustrated, which ensures the coexistence of the full-state hybrid function projective synchronization (FSHFPS) and the inverse full-state hybrid function projective synchronization (IFSHFPS) between wide classes of three-dimensional master systems and four-dimensional slave systems. In order to show the capability of the approach, a numerical example is reported, which illustrates the coexistence of FSHFPS and IFSHFPS between the incommensurate chaotic fractional-order unified system and the incommensurate hyperchaotic fractional-order Lorenz system.
\end{abstract}

MSC: $34 \mathrm{H} 10 ; 26 \mathrm{~A} 33 ; 34 \mathrm{~A} 08$

Keywords: Coexistence of synchronization types; Full-state hybrid function projective synchronization; Inverse full-state hybrid function projective synchronization; Incommensurate fractional-order systems; Non-identical systems

\section{Introduction}

Chaos synchronization refers to a process wherein two dynamical systems (master and slave systems, respectively) adjust their motion to achieve a common behavior, mainly due to a coupling or control input [1]. The issue was firstly studied in dynamical systems described by integer-order differential equations [2]. By considering the historical timeline of the topic, it can be observed that a large variety of synchronization types has been proposed [3, 4]. Among the different methods, projective synchronization provides the slave system variables consisting in scaled replicas of the master system variables [5]. Recently, the full-state hybrid projective synchronization (FSHPS) has been introduced, wherein each slave system variable synchronizes with a linear combination of master system variables. On the other hand, when the inverted scheme is implemented, i.e., each master system state synchronizes with a linear combination of slave system states, the inverse full-state hybrid projective synchronization (IFSHPS) is obtained [6]. Moreover, when the scaling factors are replaced by scaling functions, function-based hybrid synchronization schemes are obtained, i.e., the full-state hybrid function projective synchronization

(c) The Author(s) 2018. This article is distributed under the terms of the Creative Commons Attribution 4.0 International License (http://creativecommons.org/licenses/by/4.0/), which permits unrestricted use, distribution, and reproduction in any medium, provided you give appropriate credit to the original author(s) and the source, provide a link to the Creative Commons license, and indicate if changes were made. 
(FSHFPS) and the inverse full-state hybrid function projective synchronization (IFSHFPS), respectively. Note that some synchronization types may coexist in chaotic systems.

In recent years, fractional calculus has been noticed for having superior characteristics over conventional calculus in the modeling dynamics of natural phenomena [7-13]. The recent development in fractional calculus has been focused on dynamical systems in fractional sense, for example the nonlinear fractional-order systems with disturbances [14]. These systems are characterized by the fact that the order of the derivative is a noninteger number. In particular, it has been shown that may also have complex dynamics such as chaos and bifurcation [15-17]. Some efforts have been recently made devoted to the synchronization of fractional-order chaotic system $[18,19]$. It is worth noting that, differently from integer-order systems, most of the approaches for fractional-order systems are related to the synchronization of identical systems rather than non-identical systems [20]. On the other hand, referring to function-based hybrid synchronization schemes, only few schemes have been proposed to date; see [21-23]. Similar considerations hold for the coexistence of different synchronization types in fractional-order systems, given that very few attempts have been made. For example, in [24] the coexistence of some synchronization types has been illustrated, including the inverse generalized synchronization and the Q-S synchronization. However, no function-based hybrid synchronization schemes have been analyzed in [25].

Based on these considerations, this paper aims to give a further contribution to the topic by considering the coexistence of function-based hybrid synchronization in nonidentical fractional-order (incommensurate) systems characterized by different dimensions and different orders. Specifically, the paper illustrates a new theorem, which proves the coexistence of the full-state hybrid function projective synchronization (FSHFPS) and the inverse full-state hybrid function projective synchronization (IFSHFPS) between a three-dimensional master system and a four-dimensional slave system. These fractionalorder master-slave systems belong to general classes, which include several chaotic (hyperchaotic) incommensurate systems characterized by different dimensions and orders. Numerical examples of coexistence of synchronization types are illustrated, with the aim to show the effectiveness of the approach developed herein. The manuscript is organized as follows. In Sect. 2, the basic notions on fractional calculus are given, whereas in Sect. 3 the coexistence of FSHFPS and IFSHFPS for fractional-order systems is introduced. The scheme is general and the only restriction on the scaling functions is that they must be differentiable functions. In Sect. 4, by exploiting the stability of both integer-order and fractional-order linear error systems, a new theorem is illustrated, which proves the coexistence of FSHFPS and IFSHFPS between a three-dimensional incommensurate master system and a four-dimensional incommensurate slave system. Finally, in Sect. 5, numerical simulations show that the coexistence of FSHFPS and IFSHFPS is successfully achieved between the incommensurate chaotic fractional-order unified system and the incommensurate hyperchaotic fractional-order Lorenz system.

\section{Basic concepts on fractional calculus}

Both the Riemann-Liouville operator and the Caputo fractional derivative are considered through the paper. The Caputo fractional derivative is defined as follows [26]:

$$
D_{t}^{p} x(t)=J^{m-p} x^{(m)}(t) \quad \text { with } 0<p \leq 1,
$$


where $m=[p]$ (i.e., $m$ is the first integer which is not less than $p$ ), $x^{(m)}$ is the $m$ th-order derivative in the usual sense, and $J^{q}(q>0)$ is the $q$ th-order Riemann-Liouville integral operator given by

$$
J^{q} f(t)=\frac{1}{\Gamma(q)} \int_{0}^{t}(t-\tau)^{q-1} y(\tau) d \tau,
$$

where $\Gamma$ denotes Gamma function [27].

Lemma 1 ([28]) Suppose $f(t)$ has a continuous $k$ th derivative on $[0, t](k \in \mathbb{N}, t>0)$, and let $p, q>0$ such that there exist some $l \in \mathbb{N}$ with $l \leq k$ and $(p, p+q) \in[l-1, l]$. Then it results that $D_{t}^{p} D_{t}^{q} f(t)=D_{t}^{p+q} f(t)$.

Lemma 2 ([29]) The n-dimensional fractional-order linear system:

$$
D_{t}^{p} X(t)=A X(t)
$$

where $D_{t}^{p}=\left[D_{t}^{p_{1}}, D_{t}^{p_{2}}, \ldots, D_{t}^{p_{n}}\right], 0<p_{i} \leq 1,1<i \leq n$ and $A \in \mathbb{R}^{n \times n}$ is asymptotically stable if all roots $\lambda$ of the equation

$$
\operatorname{det}\left(\operatorname{diag}\left(\lambda^{M p_{1}}, \lambda^{M p_{2}}, \ldots, \lambda^{M p_{n}}\right)-A\right)=0
$$

satisfy the condition that $|\arg (\lambda)|>\frac{\pi}{2 M}$, where $M$ is the least common multiple of the denominators of $p_{i}$ 's.

\section{Coexistence of function-based hybrid synchronization schemes}

Consider the master system in the form

$$
D_{t}^{p_{i}} x_{i}(t)=f_{i}(X(t)), \quad i=1,2,3
$$

where $X(t)=\left(x_{1}(t), x_{2}(t), x_{3}(t)\right)^{T}$ is the state vector, $f_{i}: \mathbb{R}^{3} \rightarrow \mathbb{R}, 0<p_{i} \leq 1$ and $D_{t}^{p_{i}}$ is the Caputo fractional derivative of order $p_{i}$, for $i=1,2,3$. The slave system is defined as

$$
D_{t}^{q_{i}} y_{i}(t)=\sum_{j=1}^{4} b_{i j} y_{j}(t)+g_{i}(Y(t))+u_{i}, \quad i=1,2,3,4,
$$

where $Y(t)=\left(y_{1}(t), \ldots, y_{4}(t)\right)^{T}$ is the state vector, $g_{i}: \mathbb{R}^{4} \rightarrow \mathbb{R}, 0<q_{i} \leq 1, D_{t}^{q_{i}}$ is the Caputo fractional derivative of order $q_{i}$ and $u_{i}$ are synchronization controllers $(i=1,2,3,4)$.

In the following two different synchronization types are considered, where the scaling factors between the state variables of master and slave systems are replaced by scalingfunctions, indicating that function-based projective synchronization schemes are introduced.

Definition 1 The master system (5) and the slave system (6) are in full-state hybrid function projective synchronization (FSHFPS) when, for an initial condition, there exist controllers $u_{i}, 0 \leq i \leq 4$ and differentiable functions $\lambda_{i j}(t): \mathbb{R} \rightarrow \mathbb{R}(1 \leq i \leq 4 ; 1 \leq j \leq 3)$ so that the synchronization errors:

$$
e_{i}(t)=y_{i}(t)-\sum_{j=1}^{3} \lambda_{i j}(t) x_{j}(t), \quad i=1,2,3,4 .
$$

Satisfy the condition that $\lim _{t \rightarrow+\infty}\left\|e_{i}(t)\right\|=0$. 
Definition 2 The master system (5) and the slave system (6) are in inverse full-state hybrid function projective synchronization (IFSHFPS) when, for an initial condition, there exist controllers $u_{i}, 0<i \leq 4$ and differentiable functions $\mu_{i j}(t): \mathbb{R} \rightarrow \mathbb{R}(1 \leq i \leq 3 ; 1 \leq j \leq 4)$ so that the synchronization errors:

$$
e_{i}(t)=\sum_{j=1}^{4} \mu_{i j}(t) y_{j}(t)-x_{i}(t), \quad i=1,2,3 .
$$

Satisfy the condition that $\lim _{t \rightarrow+\infty}\left\|e_{i}(t)\right\|=0$.

Now the coexistence of such two different synchronization types is considered.

Definition 3 Full-state hybrid function projective synchronization (FSHFPS) and inverse full-state hybrid function projective synchronization (IFSHFPS) coexist between the master system (5) and the slave system (6), if there exist controllers $u_{i}(1 \leq i \leq 4)$ and differentiable functions $\left(\alpha_{j}(t)\right)_{1 \leq i \leq 4},\left(\beta_{j}(t)\right)_{1 \leq i \leq 3},\left(\gamma_{j}(t)\right)_{1 \leq i \leq 4}$ and $\left(\theta_{j}(t)\right)_{1 \leq i \leq 3}$, such that the following synchronization errors:

$$
\begin{aligned}
& e_{1}(t)=\sum_{j=1}^{4} \alpha_{j}(t) y_{j}(t)-x_{1}(t), \\
& e_{2}(t)=y_{2}(t)-\sum_{j=1}^{3} \beta_{j}(t) x_{j}(t), \\
& e_{3}(t)=\sum_{j=1}^{4} \gamma_{j}(t) y_{j}(t)-x_{3}(t), \\
& e_{4}(t)=y_{4}(t)-\sum_{j=1}^{3} \theta_{j}(t) x_{j}(t) .
\end{aligned}
$$

Satisfy the condition that $\lim _{t \rightarrow+\infty}\left\|e_{i}(t)\right\|=0, i=1,2,3,4$.

\section{A theorem for the coexistence of FSHFPS and IFSHFPS}

In this section the coexistence of two different function-based synchronization types is proved, i.e., FSHFPS and IFSHFPS are proved to coexist between a three-dimensional master system and a four-dimensional slave system.

The error system (9) between the master system (5) and the slave system (6) can be derived as follows:

$$
\begin{aligned}
& \dot{e}_{1}(t)=\sum_{j=1}^{4} \dot{\alpha}_{j}(t) y_{j}(t)+\sum_{j=1}^{4} \alpha_{j}(t) \dot{y}_{j}(t)-\dot{x}_{1}(t), \\
& D_{t}^{q_{2}} e_{2}(t)=D_{t}^{q_{2}} y_{2}(t)-D_{t}^{q_{2}}\left[\sum_{j=1}^{3} \beta_{j}(t) x_{j}(t)\right] \\
& \dot{e}_{3}(t)=\sum_{j=1}^{4} \dot{\gamma}_{j}(t) y_{j}(t)+\sum_{j=1}^{4} \gamma_{j}(t) \dot{y}_{j}(t)-\dot{x}_{3}(t), \\
& D_{t}^{q_{4}} e_{4}(t)=D_{t}^{q_{4}} y_{4}(t)-D_{t}^{q_{4}}\left[\sum_{j=1}^{3} \theta_{j}(t) x_{j}(t)\right]
\end{aligned}
$$


Let us suppose that the controllers $u_{i}(i=1,2,3,4)$ can be designed as follows:

$$
\begin{aligned}
& u_{1}=-\sum_{j=1}^{4} b_{1 j} y_{j}(t)-g_{1}(Y(t))+J^{1-q_{1}}\left(v_{1}\right), \\
& u_{2}=v_{2}, \\
& u_{3}=-\sum_{j=1}^{4} b_{3 j} y_{j}(t)-g_{3}(Y(t))+J^{1-q_{3}}\left(v_{3}\right), \\
& u_{4}=v_{4},
\end{aligned}
$$

where $v_{i}(1 \leq i \leq 4)$ are new controllers to be determined. By substituting Eqs. (11) into Eqs. (6), the slave system can be written as follows:

$$
D_{t}^{q_{i}} y_{i}(t)=J^{1-q_{i}}\left(v_{i}\right), \quad i=1,3,
$$

and

$$
D_{t}^{q_{i}} y_{i}(t)=\sum_{j=1}^{4} b_{i j} y_{j}(t)+g_{i}(Y(t))+v_{i}, \quad i=2,4 .
$$

By applying the Caputo fractional derivative of order $1-q_{i}(i=1,3)$ to both the left and the right sides of Eq. (12), the following result is obtained:

$$
\begin{aligned}
\dot{y}_{i}(t) & =D_{t}^{1-q_{i}}\left(D_{t}^{q_{i}} y_{i}(t)\right) \\
& =D_{t}^{1-q_{i}} J^{1-q_{i}}\left(v_{i}\right)=v_{i}, \quad i=1,3 .
\end{aligned}
$$

Note that $1-q_{i}$ satisfies $1-q_{i} \in[0,1]$. According to Lemma 1 the above statement holds. Furthermore, the error system (10) can be written as

$$
\begin{aligned}
& \dot{e}_{1}(t)=-\left|b_{11}\right| e_{1}(t)+\alpha_{1}(t) v_{1}+\alpha_{3}(t) v_{3}+R_{1}, \\
& D_{t}^{q_{2}} e_{2}(t)=-\left|b_{22}\right| e_{2}(t)+v_{2}+R_{2}, \\
& \dot{e}_{3}(t)=-\left|b_{33}\right| e_{3}(t)+\gamma_{1}(t) v_{1}+\gamma_{3}(t) v_{3}+R_{3}, \\
& D_{t}^{q_{4}} e_{4}(t)=-\left|b_{44}\right| e_{4}(t)+v_{4}+R_{4},
\end{aligned}
$$

where

$$
\begin{aligned}
& R_{1}=\left|b_{11}\right| e_{1}(t)+\sum_{j=1}^{4} \dot{\alpha}_{j}(t) y_{j}(t)+\alpha_{2}(t) \dot{y}_{2}(t)+\alpha_{4}(t) \dot{y}_{4}(t)-\dot{x}_{1}(t), \\
& R_{2}=\left|b_{22}\right| e_{2}(t)+\sum_{j=1}^{4} b_{2 j} y_{j}(t)+g_{2}(Y(t))-D_{t}^{q_{2}}\left[\sum_{j=1}^{3} \beta_{j}(t) x_{j}(t)\right], \\
& R_{3}=\left|b_{33}\right| e_{3}(t)+\sum_{j=1}^{4} \dot{\gamma}_{j}(t) y_{j}(t)+\gamma_{2}(t) \dot{y}_{2}(t)+\gamma_{4}(t) \dot{y}_{4}(t)-\dot{x}_{3}(t), \\
& R_{4}=\left|b_{44}\right| e_{4}(t)+\sum_{j=1}^{4} b_{4 j} y_{j}(t)+g_{4}(Y(t))-D_{t}^{q_{4}}\left[\sum_{j=1}^{3} \theta_{j}(t) x_{j}(t)\right] .
\end{aligned}
$$


The error system (14) can be written in the compact form as

$$
\dot{e}_{I}(t)=B_{I} e(t)+M \times V_{I}+R_{I}
$$

and

$$
D_{t}^{q} e_{I I}(t)=B_{I I} e(t)+V_{I I}+R_{I I},
$$

where

$$
\begin{aligned}
& \dot{e}_{I}(t)=\left(\dot{e}_{1}(t), \dot{e}_{3}(t)\right)^{T}, \quad D_{t}^{q} e_{I I}(t)=\left(D_{t}^{q_{2}} e_{2}(t), D_{t}^{q_{4}} e_{4}(t)\right)^{T}, \\
& B_{I}=\left(\begin{array}{cc}
-\left|b_{11}\right| & 0 \\
0 & -\left|b_{33}\right|
\end{array}\right), \quad B_{I I}=\left(\begin{array}{cc}
-\left|b_{22}\right| & 0 \\
0 & -\left|b_{44}\right|
\end{array}\right), \quad M=\left(\begin{array}{ll}
\alpha_{1}(t) & \alpha_{3}(t) \\
\gamma_{1}(t) & \gamma_{3}(t)
\end{array}\right), \\
& R_{I}=\left(R_{1}, R_{3}\right)^{T}, \quad R_{I I}=\left(R_{2}, R_{4}\right)^{T}, \quad V_{I}=\left(v_{1}, v_{3}\right)^{T} \quad \text { and } \quad V_{I I}=\left(v_{2}, v_{4}\right)^{T} .
\end{aligned}
$$

Now the following theorem can be proved.

Theorem Full-state hybrid function projective synchronization (FSHFPS) and inverse full-state hybrid function projective synchronization (IFSHFPS) coexist between the master system (5) and the slave system (6), provided that the control signals $V_{I}$ and $V_{I I}$ in the error system (15)-(16) are selected as

$$
\begin{aligned}
& V_{I}=-M^{-1} \times R_{I}, \\
& V_{I I}=-R_{I I},
\end{aligned}
$$

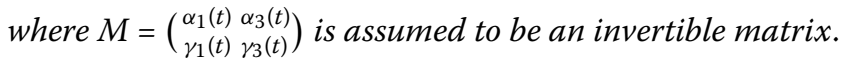

Proof By applying the control law (17) to Eq. (15), it follows that the resulting error dynamics is described by

$$
\dot{e}_{I}(t)=B_{I} e(t) .
$$

Since all the eigenvalues of $B_{I}$ have negative real part, it can be readily concluded that the integer-order linear continuous-time systems (19) is asymptotically stable, i.e., $\lim _{t \rightarrow+\infty} e_{1}(t)=\lim _{t \rightarrow+\infty} e_{3}(t)=0$. Successively, by substituting the control law (18) into Eq. (16), it follows that

$$
D_{t}^{q} e(t)=B_{I I} e(t)
$$

By computing the roots of the equation $\operatorname{det}\left(\operatorname{diag}\left(\lambda^{M q_{2}}, \lambda^{M q_{4}}\right)-B_{I I}\right)=0$, the obtained results are $\lambda_{i}=\left|b_{i i}\right|^{\frac{1}{M q_{i}}}\left(\cos \frac{\pi}{M q_{i}}+\sin \frac{\pi}{M q_{i}}\right)$, where $M$ is the least common multiple of the denominators of $q_{2}$ and $q_{4}(i=2,4)$. By computing $\arg \left(\lambda_{i}\right)$, it can be readily shown that $\left|\arg \left(\lambda_{i}\right)\right|>\frac{\pi}{2 M}$, $i=2$, 4. According to Lemma 2 , the fractional-order system (20) is asymptotically stable, indicating that $\lim _{t \rightarrow+\infty} e_{2}(t)=\lim _{t \rightarrow+\infty} e_{4}(t)=0$. Since the two error systems (19) and (20) are asymptotically stable, it can be concluded that FSHFPS and IFSHFPS coexist between the master system (5) and the slave system (6). 
(a)

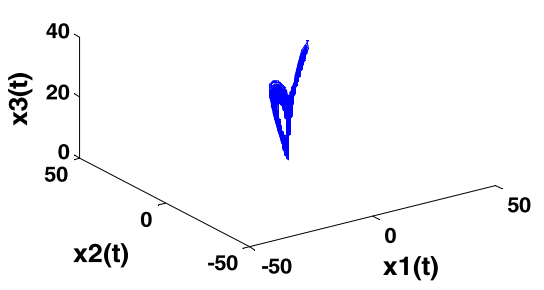

(c)

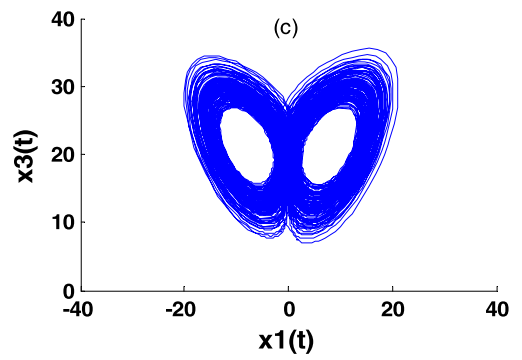

(b)

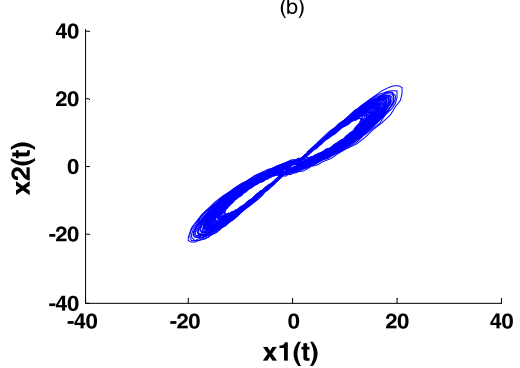

(d)

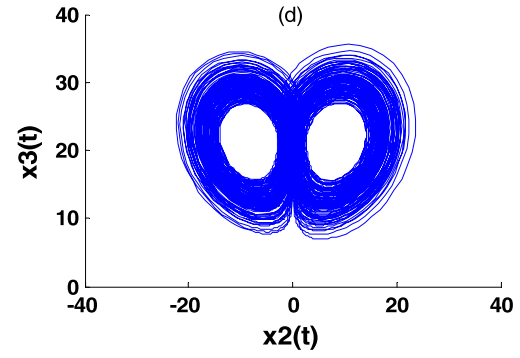

Figure 1 Chaotic attractors of the fractional-order unified system: (a) plot in the $\left(x_{1}, x_{2}, x_{3}\right)$-space; (b) plot in the $\left(x_{1}, x_{2}\right)$-plane; (c) plot in the $\left(x_{1}, x_{3}\right)$-plane; (d) plot in the $\left(x_{2}, x_{3}\right)$-plane

\section{Numerical example}

The aim of this section is to show the effectiveness of the conceived method. The selected master system is the incommensurate chaotic fractional-order unified system, whereas the slave system is the incommensurate hyperchaotic fractional-order Lorenz system. In particular, the considered master system is defined as

$$
\left\{\begin{array}{l}
D_{t}^{p_{1}} x_{1}=(25 r+10)\left(x_{2}-x_{1}\right), \\
D_{t}^{p_{2}} x_{2}=(28-35 r) x_{1}+(29 r-1) x_{2}+x_{1} x_{3} \\
D_{t}^{p_{3}} x_{3}=\frac{-(r+8)}{3} x_{3}+x_{1} x_{2} .
\end{array}\right.
$$

System (21) exhibits chaotic behavior when $\left(p_{1}, p_{2}, p_{3}\right)=(0.85,0.9,0.95)$ and $r=1$ [16]. The chaotic attractors of the incommensurate fractional-order unified system are shown in Fig. 1.

The slave system is the fractional-order Lorenz system described by

$$
\left\{\begin{array}{l}
D_{t}^{q_{1}} y_{1}=a\left(y_{2}-y_{1}\right)+y_{4}+u_{1}, \\
D_{t}^{q_{2}} y_{2}=c y_{1}-y_{2}-y_{1} y_{3}+u_{2}, \\
D_{t}^{q_{3}} y_{3}=-b y_{3}+y_{1} y_{2}+u_{3}, \\
D_{t}^{q_{4}} y_{4}=d y_{4}+y_{2} y_{3}+u_{4},
\end{array}\right.
$$

where $U=\left(u_{1}, u_{2}, u_{3}, u_{4}\right)^{T}$ is the vector controller. This system exhibits hyperchaotic behavior when $\left(u_{1}, u_{2}, u_{3}, u_{4}\right)=(0,0,0,0),\left(q_{1}, q_{2}, q_{3}, q_{4}\right)=(0.94,0.96,0.97,0.99)$ and $(a, b$, $c, d)=\left(10, \frac{8}{3}, 28,-1\right)[17]$. Some plots of the hyperchaotic Lorenz attractor are shown in Fig. 2. 
(a)

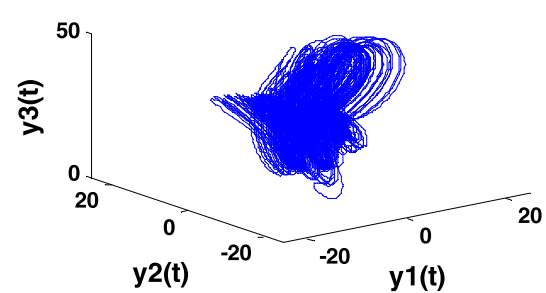

(c)

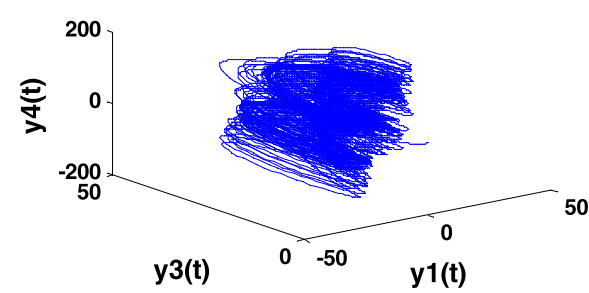

(b)

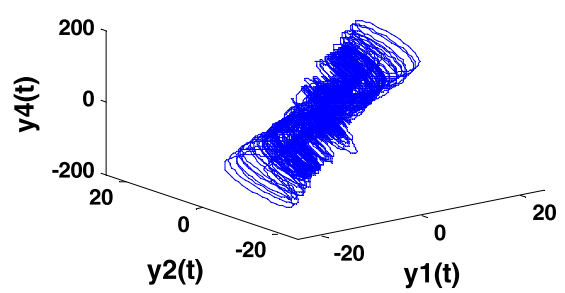

(d)

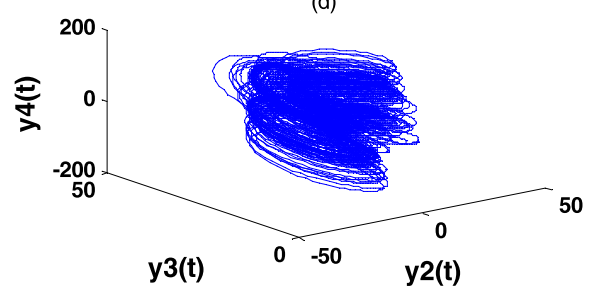

Figure 2 Hyperchaotic attractors of the fractional-order Lorenz system: (a) plot in the $\left(y_{1}, y_{2}, y_{3}\right)$-space; (b) plot in the $\left(y_{1}, y_{2}, y_{4}\right)$-space; (c) plot in the $\left(y_{1}, y_{3}, y_{4}\right)$-space; (d) plot in the $\left(y_{2}, y_{3}, y_{4}\right)$-space

By putting system (22) in the form (6), we have

$$
B=\left(b_{i j}\right)=\left(\begin{array}{cccc}
-10 & 10 & 0 & 1 \\
28 & -1 & 0 & 0 \\
0 & 0 & -8 / 3 & 0 \\
0 & 0 & 0 & -1
\end{array}\right), \quad\left(g_{i}\right)_{1 \leq i \leq 4}=\left(\begin{array}{c}
0 \\
-y_{1} y_{3} \\
y_{1} y_{2} \\
y_{2} y_{3}
\end{array}\right)
$$

According to the conceived approach, FSHFPS and IFSHFPS coexist between the master system (21) and the slave system (22) if the following synchronization errors:

$$
\begin{aligned}
& e_{1}=\sum_{j=1}^{4} \alpha_{j}(t) y_{j}-x_{1}, \\
& e_{2}=y_{2}-\sum_{j=1}^{3} \beta_{j}(t) x_{j}, \\
& e_{3}=\sum_{j=1}^{4} \gamma_{j}(t) y_{j}-x_{3}, \\
& e_{4}=y_{4}-\sum_{j=1}^{3} \theta_{j}(t) x_{j},
\end{aligned}
$$

asymptotically approach zero, where the functions $\left(\alpha_{j}(t)\right)_{1 \leq i \leq 4},\left(\beta_{j}(t)\right)_{1 \leq i \leq 3},\left(\gamma_{j}(t)\right)_{1 \leq i \leq 4}$ and $\left(\theta_{j}(t)\right)_{1 \leq i \leq 3}$ have been selected as

$$
\begin{aligned}
& \alpha_{1}(t)=t^{2}+1, \quad \alpha_{2}(t)=t, \quad \alpha_{3}(t)=0, \quad \alpha_{4}(t)=-3, \\
& \beta_{1}(t)=0, \quad \beta_{2}(t)=2 t, \quad \beta_{3}(t)=0,
\end{aligned}
$$




$$
\begin{aligned}
& \gamma_{1}(t)=0, \quad \gamma_{2}(t)=t, \quad \gamma_{3}(t)=\exp (t), \quad \gamma_{4}(t)=0, \\
& \theta_{1}(t)=t^{2}+1, \quad \theta_{2}(t)=0, \quad \theta_{3}(t)=t .
\end{aligned}
$$

By applying the theorem illustrated in the previous section, we have

$$
\begin{aligned}
& B_{I}=\left(\begin{array}{cc}
-10 & 0 \\
0 & -\frac{8}{3}
\end{array}\right), \quad B_{I I}=\left(\begin{array}{cc}
-1 & 0 \\
0 & -1
\end{array}\right), \\
& M=\left(\begin{array}{cc}
t^{2}+1 & 0 \\
0 & \exp (t)
\end{array}\right), \quad M^{-1}=\left(\begin{array}{cc}
\frac{1}{t^{2}+1} & 0 \\
0 & \exp (-t)
\end{array}\right) .
\end{aligned}
$$

As a consequence, the controllers $u_{i}(1 \leq i \leq 4)$ in (22) can be written as

$$
\begin{aligned}
& u_{1}=-a\left(y_{2}-y_{1}\right)-y_{4}+J^{0.06}\left[\frac{-1}{t^{2}+1}\left(10 e_{1}(t)+2 t y_{1}+y_{2}+t \dot{y}_{2}-3 \dot{y}_{4}-\dot{x}_{1}\right)\right], \\
& u_{2}=-e_{2}-c y_{1}+y_{2}+y_{1} y_{3}+2 D^{0.96}\left[t x_{2}\right] \\
& u_{3}=b y_{3}-y_{1} y_{2}+J^{0.03}\left[-\exp (-t)\left(\frac{8}{3} e_{3}+y_{2}+\exp (t) y_{3}+t \dot{y}_{2}-\dot{x}_{3}\right)\right] \\
& u_{4}=-e_{4}-d y_{4}-y_{2} y_{3}+D^{0.99}\left[\left(t^{2}+1\right) x_{1}+t x_{3}\right]
\end{aligned}
$$

indicating that the error sub-system (15) can be written in the form

$$
\begin{aligned}
& \dot{e}_{1}(t)=-10 e_{1}(t), \\
& \dot{e}_{3}(t)=-\frac{8}{3} e_{3}(t),
\end{aligned}
$$

whereas the error sub-system (16) can be written as

$$
\begin{aligned}
& D_{t}^{0.96} e_{2}(t)=-e_{2}(t), \\
& D_{t}^{0.99} e_{4}(t)=-e_{4}(t) .
\end{aligned}
$$

Numerical simulations have been carried out for solving systems (25) and (26). In particular, the fourth-order Runge-Kutta integration method has been applied to the integerorder system (25), whereas the fractional Euler integration method has been used for solving the incommensurate fractional-order system (26). The initial states of the master system and the slave system are $\left(x_{1}(0), x_{2}(0), x_{3}(0)\right)=(-1,1,10)$ and $\left(y_{1}(0), y_{2}(0), y_{3}(0)\right.$, $\left.y_{4}(0)\right)=(2,-2,1,-1)$, respectively. The initial states of the error system $(25)-(26)$ are $\left(e_{1}(0), e_{2}(0), e_{3}(0), e_{4}(0)\right)=(1,-2,-10,-1)$. Figures 3 and 4 display the synchronization errors between the master system (21) and the slave system (22), indicating that the coexistence of full-state hybrid function projective synchronization (FSHFPS) and inverse fullstate hybrid function projective synchronization (IFSHFPS) is effectively achieved.

\section{Conclusion and future work}

When analyzing the synchronization of fractional-order chaotic systems, an interesting phenomenon that may occur is the coexistence of some synchronization types. 


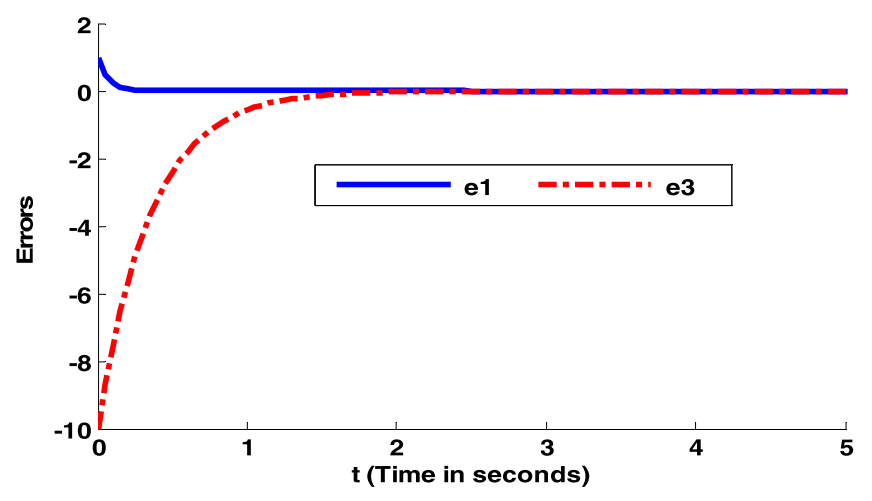

Figure 3 Time evolution of the errors $e_{1}(t)$ and $e_{3}(t)$

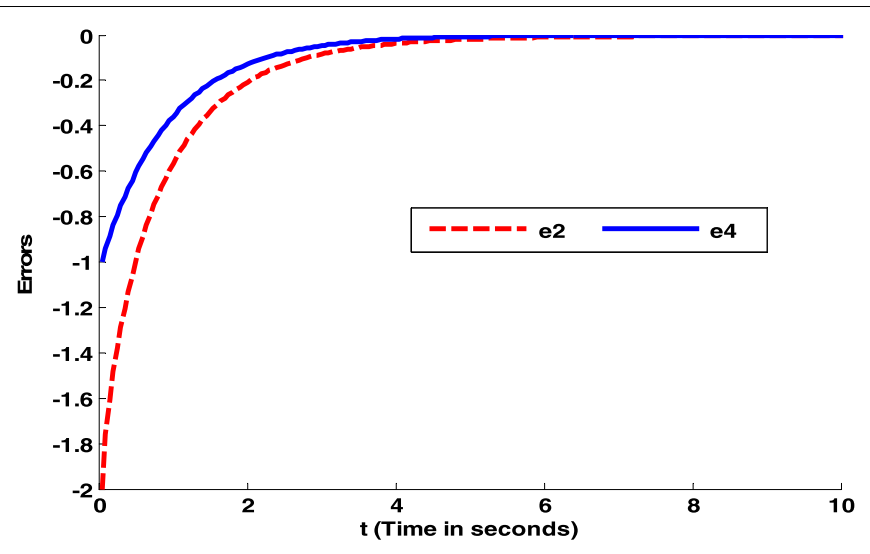

Figure 4 Time evolution of the errors $e_{2}(t)$ and $e_{4}(t)$

Based on these considerations, this paper has presented new results related to the coexistence of function-based hybrid synchronization types between non-identical incommensurate fractional-order systems characterized by different dimensions and orders. Specifically, the manuscript has proposed a new theorem, which ensures the coexistence of FSHFPS and IFSHFPS between a three-dimensional master system and a fourdimensional slave system. Note that the approach developed herein enables to prove the coexistence of FSHFPS and IFSHFPS in several cases. Specifically, the approach can be applied to: (i) wide classes of chaotic (hyperchaotic) fractional-order master-slave systems; (ii) non-identical incommensurate fractional-order systems with different dimensions; (iii) schemes wherein the scaling factor of the linear combination can be any arbitrary differentiable function. A numerical example, describing the coexistence of FSHFPS and IFSHFPS between the incommensurate chaotic fractional-order unified system and the incommensurate hyperchaotic fractional-order Lorenz system, has clearly highlighted the effectiveness of the approach proposed herein.

Finally, we would make some comments on future developments of the present work. In particular, we are conscious that circuit implementations of synchronization schemes are an important issue. For this reason, we are preparing a forthcoming paper where all the details related to the circuit implementation of the conceived synchronization scheme will be provided. Further developments and extended analysis related to the application 
of the new hybrid synchronization to secure communication systems and new complex fractional schemes of synchronization will be investigated in a future work.

\author{
Acknowledgements \\ The authors acknowledge Prof. GuanRong Chen, Department of Electronic Engineering, City University of Hong Kong for \\ suggesting many helpful references.
}

\title{
Funding
}

The author Xiong Wang was supported by the National Natural Science Foundation of China (No. 61601306) and Shenzhen Overseas High Level Talent Peacock Project Fund (No. 20150215145C).

\section{Competing interests}

The authors declare that they have no competing interests.

\section{Authors' contributions}

AO and VTP made substantial contributions to conception and design. AO, XW, VTP, GG and TZ carried out results interpretation and analysis. XW, GG and TZ helped to evaluate, revise and edit the manuscript. AO and VTP drafted the article. All authors read and approved the final manuscript.

\section{Author details}

${ }^{1}$ Department of Mathematics, University of Tebessa, Tebessa, Algeria. ${ }^{2}$ Dipartimento Ingegneria Innovazione, Università del Salento, Lecce, Italy. ${ }^{3}$ Institute for Advanced Study, Shenzhen University, Shenzhen, China. ${ }^{4}$ Department of Material Sciences, University of Tebessa, Tebessa, Algeria. ${ }^{5}$ Modeling Evolutionary Algorithms Simulation and Artificial Intelligence, Faculty of Electrical \& Electronics Engineering, Ton Duc Thang University, Ho Chi Minh City, Vietnam.

\section{Publisher's Note}

Springer Nature remains neutral with regard to jurisdictional claims in published maps and institutional affiliations.

Received: 7 March 2018 Accepted: 21 August 2018 Published online: 04 September 2018

\section{References}

1. Ott, E.: Chaos in Dynamical Systems. Cambridge University Press, Cambridge (2002)

2. Pecora, L.M., Carroll, T.L.: Synchronization in chaotic systems. Phys. Rev. Lett. 64(8), 821-824 (1990)

3. Aziz-Alaoui, M.A.: Synchronization of chaos. In: Encyclopedia of Mathematical Physics, vol. 5, pp. $213-226$ (2006)

4. Manieri, R., Rehacek, J.: Projective synchronization in three-dimensional chaotic systems. Phys. Rev. Lett. 82(15), 3042-3045 (1999)

5. Hu, M., Xu, Z., Zhang, R.: Full state hybrid projective synchronization in continuous-time chaotic (hyperchaotic) systems. Commun. Nonlinear Sci. Numer. Simul. 13(2), 456-464 (2008)

6. Ouannas, A., Grassi, G.: Inverse full state hybrid projective synchronization for chaotic maps with different dimensions. Chin. Phys. B 25(9), 090503 (2016)

7. Yang, X.J.: Fractional derivatives of constant and variable orders applied to anomalous relaxation models in heat transfer problems. Therm. Sci. 21(3), 1161-1171 (2017)

8. Yang, X.J., Tenriero Machado, J.A., Baleanu, D.: Exact traveling-wave solution for local fractional Boussinesq equation in fractional domain. Fractals 25, 1740006 (2017)

9. Yang, X.J., Gao, F., Srivastava, H.M.: A new computational approach for solving nonlinear local fractional PDEs. J. Comput. Appl. Math. 339, 285-296 (2018)

10. Wu, G.C., Baleanu, D., Huang, L.L.: Novel Mittag-Leffler stability of linear fractional delay difference equations with impulse. Appl. Math. Lett. 82, 71-78 (2018)

11. Guo, Y.: Exponential stability analysis of traveling waves solutions for nonlinear delayed cellular neural networks. Dyn. Syst. 32, 490-503 (2017)

12. Kucche, K.D., Trujillo, J.J.: Theory of system of nonlinear fractional differential equations. Prog. Fract. Differ. Appl. 3, 7-18 (2017)

13. Khaldi, R., Guezane-Lakoud, A.: Upper and lower solutions method for higher order boundary value problems. Prog. Fract. Differ. Appl. 3, 53-57 (2017)

14. Jajarmi, A., Hajipour, M., Mohammadzadeh, E., Baleanu, D.: A new approach for the nonlinear fractional optimal control problems with external persistent disturbances. J. Franklin Inst. 335(9), 3938-3967 (2018)

15. Hajipour, M., Jajarmi, A., Baleanu, D.: An efficient nonstandard finite difference scheme for a class of fractional chaotic systems. J. Comput. Nonlinear Dyn. 13(2), 021013 (2018)

16. Deng, W., Li, C.: The evolution of chaotic dynamics for fractional unified system. Phys. Lett. A 372, $401-407$ (2008)

17. Li, T.Z., Wang, Y., Luo, M.K.: Control of fractional chaotic and hyperchaotic systems based on a fractional order controller. Chin. Phys. B 23(8), 080501 (2014)

18. Fan, Y., Huang, X., Wang, Z., Lia, Y.: Improved quasi-synchronization criteria for delayed fractional-order memristor-based neural networks via linear feedback control. Neurocomputing 306, 68-79 (2018)

19. Huang, X., Fan, Y., Jia, J., Wang, Z., Li, Y.: Quasi-synchronisation of fractional-order memristor-based neural networks with parameter mismatches. IET Control Theory Appl. 11(14), 2317-2327 (2017)

20. Zhang, F., Chen, G., Li, C., Kurths, J.: Chaos synchronization in fractional differential systems. Philos. Trans. R. Soc. A 371, $20120155(2013)$

21. Sha, W., Yu, Y.G., Hu, W., Rahmani, A.: Function projective lag synchronization of fractional-order chaotic systems. Chin. Phys. B 23(4), 040502 (2014) 
22. Yadav, V.K., Srikanth, N., Das, S.: Dual function projective synchronization of fractional order complex chaotic systems. Optik 127, 10527-10538 (2016)

23. Ouannas, A., Grassi, G., Ziar, T., Odibat, Z:: On a function projective synchronization scheme for non-identical fractional-order chaotic (hyperchaotic) systems with different dimensions and orders. Optik 136, 513-523 (2017)

24. Ouannas, A., Odibat, Z., Hayat, T.: Fractional analysis of co-existence of some types of chaos synchronization. Chaos Solitons Fractals 105, 215-223 (2017)

25. Ouannas, A., Wang, X., Pham, V.T., Grassi, G., Ziar, T.: Co-existence of some synchronization types between non-identical commensurate and incommensurate fractional-order chaotic systems with different dimensions. Adv. Differ. Equ. 2018, 21 (2018)

26. Podlubny, l.: Fractional Differential Equations. Academic Press, New York (1999)

27. Samko, S.G., Klibas, A.A., Marichev, O.I.: Fractional Integrals and Derivatives: Theory and Applications. Gordon \& Breach, Amsterdam (1993)

28. Si, G., Sun, Z., Zhang, Y., Chen, W.: Projective synchronization of different fractional-order chaotic systems with non-identical orders. Nonlinear Anal., Real World Appl. 13, 1761-1771 (2012)

29. Matignon, D.: Stability results of fractional differential equations with applications to control processing. In: IMACS, IEEE-SMC, Lille, France (1996)

\section{Submit your manuscript to a SpringerOpen ${ }^{\circ}$ journal and benefit from:}

- Convenient online submission

- Rigorous peer review

- Open access: articles freely available online

- High visibility within the field

- Retaining the copyright to your article

Submit your next manuscript at $\boldsymbol{\nabla}$ springeropen.com 